\title{
Detection of functional activity in brain white matter using fiber architecture informed synchrony mapping
}

\author{
Yu Zhao ${ }^{1,2 *}$, Yurui Gao ${ }^{1,3}$, Zhongliang Zu',2, Muwei Li1, 2, Kurt G. Schilling ${ }^{1,3}$, Adam W. \\ Anderson ${ }^{1,2,3}$, Zhaohua Ding ${ }^{1,3,6^{*}}$, John C. Gore ${ }^{1,2,3,4,5}$
}

\begin{abstract}
A general linear model is widely used for analyzing fMRI data, in which the blood oxygenation-level dependent (BOLD) signals in gray matter (GM) evoked in response to neural stimulation are modeled by convolving the time course of the expected neural activity with a canonical hemodynamic response function (HRF) obtained a priori. The maps of brain activity produced reflect the magnitude of local BOLD responses. However, detecting BOLD signals in white matter (WM) is more challenging as the BOLD signals are weaker and the HRF is different, and may vary more across the brain. Here we propose a model-free approach to detect changes in BOLD signals in WM by measuring task-evoked increases of BOLD signal synchrony in WM fibers. The proposed approach relies on a simple assumption that, in response to a functional task, BOLD signals in relevant fibers are modulated by stimulus-evoked neural activity and thereby show greater synchrony than when measured in a resting state, even if their magnitudes do not change significantly. This approach is implemented in two technical stages. First, for each voxel a fiberarchitecture-informed spatial window is created with orientation distribution functions constructed from diffusion imaging data. This provides the basis for defining neighborhoods in WM that share similar local fiber architectures. Second, a modified principal component analysis (PCA) is used to estimate the synchrony of BOLD signals in each spatial window. The proposed approach is validated using a 3T fMRI dataset from the Human Connectome Project (HCP) at a group level. The results demonstrate that neural activity can be reliably detected as increases in $\mathrm{fMRI}$ signal synchrony within WM fibers that are engaged in a task with high sensitivities and reproducibility.
\end{abstract}

Key words-Functional MRI, white matter, activation mapping, graph signal processing, synchrony mapping.

${ }^{1}$ Vanderbilt University Institute of Imaging Science, United States of America.

2 Department of Radiology and Radiological Sciences, Vanderbilt University Medical Center, United States of America.

3 Department of Biomedical Engineering, Vanderbilt University, United States of America.

4 Department of Molecular Physiology and Biophysics, Vanderbilt University, United States of America.

5

${ }^{5}$ Department of Physics and Astronomy, Vanderbilt University, United States of America.

6 Department of Electrical and Computer Engineering, Vanderbilt University, United States of America.

The corresponding authors: Zhaohua Ding and Yu Zhao (zhaohua.ding@vanderbilt.edu and yu.zhao.1@vumc.org). 


\section{Introduction}

$\mathrm{F}$ UCTIONAL MRI (fMRI) based on blood oxygenation level-dependent (BOLD) signals is well established for detecting and mapping neural activities in brain gray matter (GM). However, there are relatively few corresponding reports of fMRI studies of white matter (WM), partly due to continuing uncertainties regarding the source of BOLD signals therein ${ }^{1,2}$ as well as technical challenges in their reliable detection. Nonetheless, the existence and potential functional relevance of BOLD signals in WM have been validated by a growing number of recent studies, (see reviews in ${ }^{3,4}$ ). These have been partially motivated by the fact that WM comprises about half of the brain parenchyma and plays an important role as the communication system of the brain, and so the functional properties of WM should be included in any complete model of brain organization. In the emerging field of WM fMRI, analysis methods established for GM have been adopted and applied, including measures of function connectivity ${ }^{5-7}$ and spectral analysis 8-11, and these have shown the potential of characterizing selected features of WM in neurodegenerative and other disorders.

The standard approach that has been widely used in fMRI studies of GM uses a general linear model (GLM) to detect activations that correspond to a change in the local BOLD signal magnitude, but this may not be appropriate for WM as suggested by previous reports ${ }^{12}$. The GLM assumes that the BOLD response to stimulation may be treated as a linear shiftinvariant system so that the BOLD signals can be modeled by convolving the time course of neural activities with a hemodynamic response function (HRF). The GLM assumes that evoked neural activations are synchronous with the timings of presented stimuli or tasks that trigger hemodynamic responses ${ }^{13}$ and correspond to a change in signal magnitude. This assumption may not be valid in all situations, especially in WM because it ignores intrinsic brain activities that pre-exist in the background and are unrelated to extrinsic stimuli. Such intrinsic brain activities were considered noise before they were found to predict spatial patterns of functional connectivity (i.e., functional networks) in a resting state ${ }^{14,15}$. A voxel in WM contains a large number of axons that connect neurons in different positions in GM 16,17 and these may produce asynchronous neuronal signals so that fMRI signals measured in a WM voxel may be more complex than those in GM and thus inconsistent with stimuli in tasks. A further assumption in the GLM is that the HRF keeps fixed for all positions, as implemented in SPM ${ }^{13}$. This assumption is not valid in all WM as previous studies have shown that HRFs measured in WM exhibit substantial regional variations ${ }^{12,18}$. A potential solution to this issue is to use variants of a GLM that allow HRFs to adapt spatially. However, potential mismodeling of HRF in such approaches may prevent accurate activation mapping and reduce sensitivity. Furthermore, fMRI signal fluctuations in WM are substantially lower than those in GM ${ }^{19}$ due to the significantly lower blood volume in WM compared to GM ${ }^{1,20}$ and the resulting lower BOLD signal-noise-ratio poses additional challenges to the analysis of WM.

A model-free approach, without the assumptions mentioned above, that produces robust detection of activity-related BOLD signals in WM is thus needed. An alternative to the GLM that incorporates the temporal coherence of a voxel with respect to its neighbors may provide a means of activation mapping without modeling the BOLD signal or assuming local changes in signal magnitude. This approach is inspired by previous findings that the regional homogeneity (ReHo) of fMRI signals in GM is modulated by stimuli presented in tasks ${ }^{21-23}$. The ReHo approach relies on the fact that voxels within a GM volume at a macroscopic scale constitute a functional unit in which the synchrony of inter-voxel time courses can be enhanced under functional loading. In contrast to GM, anatomical structures of the WM feature complex interwoven networks, in which fibrous tissues constitute elongated functional units for neural signal transmission and thus show structural anisotropy. Evidently, brain functional processes embedded in such an anisotropic substrate should also exhibit anisotropic spatial profiles. This has been demonstrated by previous studies of BOLD signals in WM ${ }^{24-26}$, which reported structure-specific temporal correlations along WM tracts in a resting state that become more pronounced in relevant structures under a task loading. Given the relationships between local fiber architectures and WM function, we propose a new approach for mapping activity-related BOLD signals in WM that incorporates a metric of BOLD signal synchrony. This approach introduces two technical innovations. First, an adaptive spatial window is designed for each voxel to extract and weight time courses associated with a fiber-architectureinformed neighborhood of the voxel. The neighborhood is determined using diffusion orientation distribution functions (ODFs) obtained from high angular resolution diffusion imaging (HARDI) data. A similar concept was proposed in a previous report that used anisotropic spatial filtering to enhance noisy BOLD contrasts in $\mathrm{WM}^{27}$. Second, a modified principal component analysis (PCA) is applied to time courses of fMRI data to estimate the synchrony of time courses within the fiberarchitecture-informed window of each voxel. Taken together, the proposed approach overcomes the intrinsic limitations of GLM based analyses of BOLD signals in WM.

In the following, the proposed model-free mapping of neural activations is illustrated using a $3 \mathrm{~T}$ fMRI dataset from the Human Connectome Project (HCP) at a group level. First, spatial patterns within activation maps are evaluated by reference to anatomical structures of WM fibers derived from diffusion MRI. Then, the influence of task loading on activation are investigated by comparing activation maps derived from motor-task fMRI data with those acquired in a resting-state. Motor-task enhanced activations in WM are demonstrated in WM regions related to the primary motor cortex, or M1, which are determined by WM fiber tracking with seed points in M1. Furthermore, the sensitivity, specificity and reproducibility of the proposed method are assessed statistically based on fibertracking-based parcellations of task-related and unrelated WM regions.

\section{Methods}

The proposed approach consists of two major steps implemented on a voxel-by-voxel basis (Fig. 1). First, a fiberarchitecture-informed spatial window is created for each voxel using ODFs constructed from HARDI data. Second, a modified 


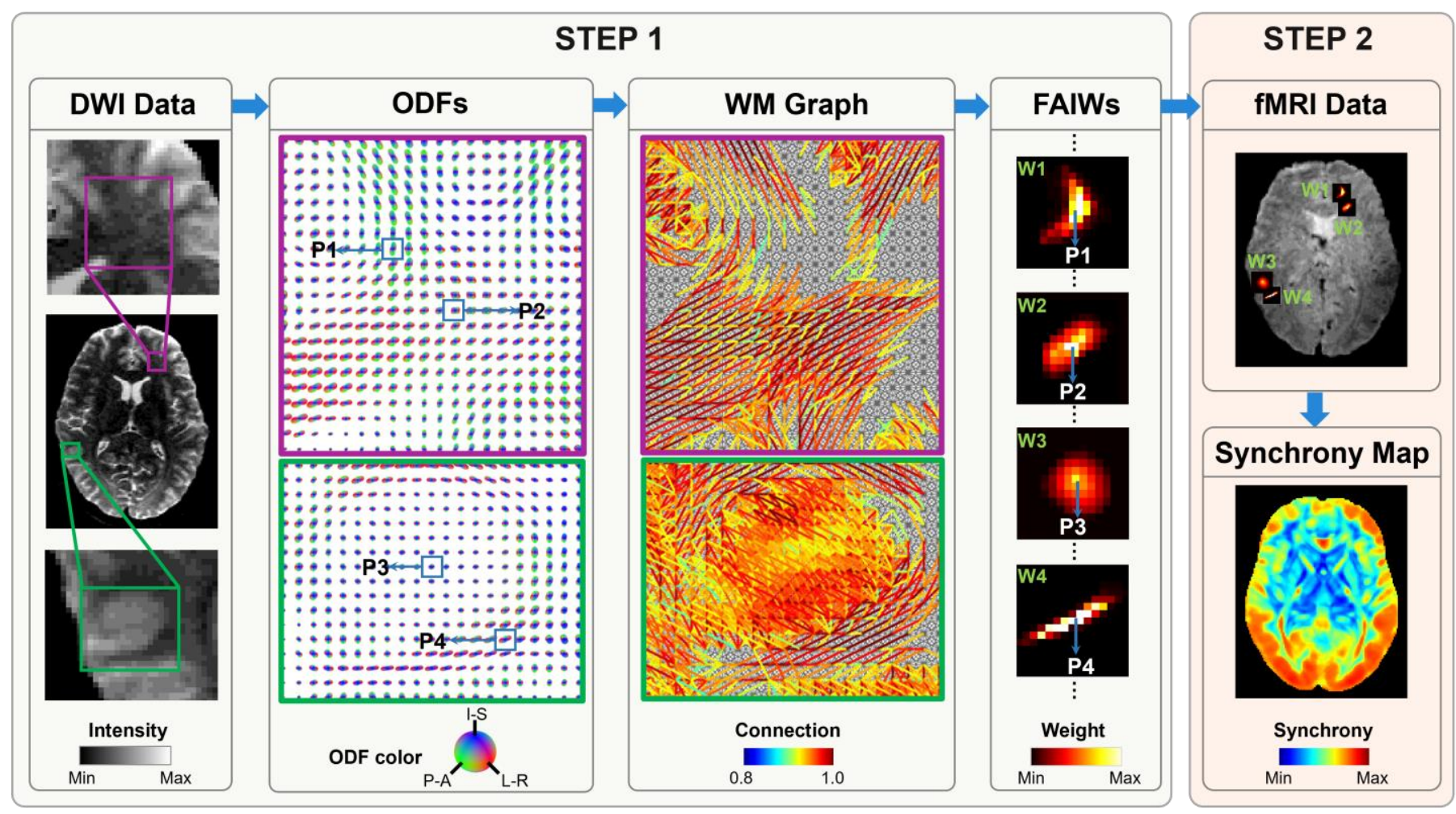

Fig. 1. Schematic of fiber architecture informed synchrony mapping in human white matter (WM). The algorithm of the proposed approach consists of two major steps. First, a spatial window is created for each voxel based on local fiber architectures. In this step, orientation distribution functions (ODFs) constructed from diffusion MRI data provide the information on fiber architectures, which are then used to generate a topological graph for WM. In this graph, every vertex corresponds to a voxel in MR images, with weighted connections to its neighbors. The edge weights are determined by the coherence between the directions of diffusion and the orientation of the graph edges. Then, diffusion on the graph with a point source located at each vertex is simulated to produce a diffusion profile that can be further used as a fiber-architecture-informed window (FAIWs). Here, typical FAIWs created for four voxels (P1, P2, P3 and P4) demonstrate that they are adaptive to local fiber architectures. Second, a modified PCA is implemented to estimate the synchrony of fMRI time courses based on the FAIWs from the first step to yield activation maps. In the modified PCA, the FAIWs emphasize the time courses associated to voxels near the central position with high weights, which ensures the spatial specificity of the synchrony estimate.

PCA is used to estimate the synchrony of fMRI time courses based on this spatial window. Detailed algorithms and procedures of the proposed approach are described as follows.

\section{A. Design of fiber-architecture-informed spatial window}

Principles from the recently emerging field of graph signal processing (GSP) ${ }^{28,29}$ provide an elegant framework to design the fiber-architecture-informed spatial window. Based on the GSP framework, we analyze fMRI time courses at a discrete set of positions, which form a set of vertices of a topological graph, in such a way that underlying constraints from fiber architectures can be accounted for by edges of the graph. Here, we denote a graph as $\mathbf{G}=\{\mathbf{V}, \mathbf{E}, \mathbf{W}\}$ as usual, which consists of a finite set of vertices $\mathbf{V}$ with $|\mathbf{V}|=\mathrm{N}$, a set of edges $\mathbf{E}$, and a weighted connection matrix $\mathbf{W} . \mathbf{W}_{i, j}$ represents the connection strength of edge $\boldsymbol{e}=(i, j)$ that connects vertices $i$ and $j$. On the vertices of the graph, we define a vector $\mathbf{f} \in \mathbb{R}^{N}$ to represent a set of auxiliary quantities to be used to generate spatial windows, where the $i$-th component of the vector, $\mathbf{f}(i)$, represents the value at the $i$-th vertex in $\mathbf{V}$.

For this study, voxel-wise graphs are constructed using ODFs derived from HARDI data, which have been well described in previous reports ${ }^{27,30}$. In each graph, every vertex corresponds to a voxel in 3D MR images with weighted connections to its neighbors. The connection weight of any two neighboring voxels depends on the coherence of diffusion orientations between the two voxels. As a result, two neighboring voxels residing in the same fiber tract have relative larger weight than those in different fiber tracts.

Building on the topological properties of the fiberarchitecture-informed graph, a spatial window is created for each vertex/voxel by simulating diffusion on the graph with point sources located at each vertex. A kernel is defined for the diffusion process in the spectral domain, which is derived from a Laplacian matrix defined in GSP. Specifically, a Laplacian matrix of $\mathbf{G}$ is defined as $\mathbf{L}=\mathbf{D}-\mathbf{W}$, where $\mathbf{D}$ denotes the graph's degree matrix, which is diagonal with elements $\boldsymbol{d}_{\boldsymbol{i}, \boldsymbol{i}}=$ $\sum_{j} \mathbf{w}_{i, j}$; the graph operator $\mathbf{L}$ is a real symmetric matrix, and thus has a complete set of orthonormal eigenvectors $\left\{\boldsymbol{u}_{\ell}\right\}_{\ell=1,2, \ldots, N}$ and associated eigenvalues $\left\{\boldsymbol{\lambda}_{\ell}\right\}_{\ell=1,2, \ldots, N}$ satisfying $\mathbf{L} \boldsymbol{u}_{\ell}=\lambda_{\ell} \boldsymbol{u}_{\ell}$. With these eigenvectors, the graph signal $\mathbf{f}$ can be transformed into the spectral domain,

$$
\hat{\mathbf{f}}(\ell)=\sum_{i=1}^{N} \boldsymbol{u}_{\ell}^{\mathrm{T}}(i) \mathbf{f}(i)=\boldsymbol{u}_{\ell}^{\mathrm{T}} \mathbf{f}, \ell=1,2, \ldots, N
$$


This spectral representation allows an exact reconstruction, i.e., the signal $\mathbf{f}$ can be recovered as

$$
\mathbf{f}=\sum_{\ell=1}^{N} \hat{\mathbf{f}}(\ell) \boldsymbol{u}_{\ell}
$$

In the graph spectral domain, the kernel of diffusion ${ }^{31}$ can be defined as

$$
K(\lambda)=e^{-\tau \lambda}
$$

where $\tau$ is a free parameter determining the spatial extent of diffusion on a graph, and it can be intuitively treated as the duration of diffusion. Given an initial point source $\mathbf{f}_{0}(t=0)$, the transient diffusion profile at time point $\mathrm{t}$ can be derived as

$$
\mathbf{f}(t)=\sum_{\ell=1}^{N} K\left(\lambda_{\ell}\right) \hat{\mathbf{f}}_{0}(\ell) \boldsymbol{u}_{\ell}=\sum_{\ell=1}^{N} e^{-\lambda_{\ell} t} \boldsymbol{u}_{\ell}^{\mathrm{T}} \mathbf{f}_{0} \boldsymbol{u}_{\ell}
$$

To create a spatial window centered at a voxel $i$ we define this point source by setting the initial profile $\mathbf{f}_{0}$ to be equal to 1 at the position $i$ and 0 otherwise. The transient profile $\mathbf{f}(t)$ at the time point $t$ is further processed to yield the fiber-architectureinformed spatial window, the width of which can be adjusted by the parameter $\tau$. To be specific, the window is defined by taking voxels with $M$ largest values of $\mathbf{f}(t)$, which is subsequently normalized with their summation,

$$
\mathbf{F}_{i}(j)=\mathbf{f}(j, t) / \sum_{j=1}^{M} \mathbf{f}(j, t)
$$

In this study, the largest parameter $M$ is taken under an empirical constraint $\sum_{j=1}^{M} \mathbf{f}(j, t)>0.95$, wherein small values in the diffusion profile of each voxel are removed to improve computational efficiency.

\section{B. PCA based on fiber-architecture-informed window}

In previous reports, the synchrony of fMRI time-courses in a parcellated region have been quantified using PCA ${ }^{32}$, wherein the variance explained by the first principal component gauges the extent of synchrony. Here, the conventional PCA is modified to allow the integration of fiber architecture information, referred to as a modified PCA based on a fiberarchitecture-informed window (FAIW-PCA). Basically, the raw fMRI time courses are first normalized with respect to the mean values and the standard deviations. Then, within each window $\mathrm{F}_{i}$ centered at the position $\mathrm{i}$, the normalized time courses are rearranged into a matrix $(\mathrm{X})$ of dimension $\mathrm{T} \times \mathrm{M}$, where $T$ denotes the number of time frames and $M$ the number of voxels within the window. We denote a time course in $\mathrm{X}$ as $\boldsymbol{x}_{i}$, the i-th column in the matrix X, and formulate the FAIWPCA as an optimization problem, where the first principal component $v_{1}$ can be obtained by

$$
\boldsymbol{v}_{1}=\arg \max _{\|\boldsymbol{v}\|=1}\left\{\sum_{j=1}^{M} \mathbf{F}_{i}(j) \cdot\left(\boldsymbol{x}_{j} \cdot \boldsymbol{v}\right)^{2}\right\} .
$$

Note that, without the weights $\mathbf{F}_{\boldsymbol{i}}(j)$, this optimization problem will degenerate into a conventional PCA. Here, $\mathbf{F}_{i}(j)$ is applied in the optimization such that the time courses associated with the voxels near the center position are emphasized more in the first principal component obtained, which emphasizes the spatial specificity of the synchrony estimate. We further write this optimization in matrix form,

$$
\boldsymbol{v}_{1}=\arg \max _{\|v\|=1}\left\{\boldsymbol{v}^{T} \mathrm{X}^{T} \mathrm{QX} \boldsymbol{v}\right\}
$$

where the matrix $Q=\operatorname{diag}\left[\left(\mathbf{F}_{\boldsymbol{i}}(1), \mathbf{F}_{\boldsymbol{i}}(2), \ldots, \mathbf{F}_{\boldsymbol{i}}(M)\right]\right.$. With simple algebraic manipulations, Equation 7 can be written as

$$
v_{1}=\arg \max \left\{\frac{v^{T} \mathrm{H} v}{v^{T} v}\right\}
$$

where $\mathrm{H}=\mathrm{X}^{T} \mathrm{QX}$. $\mathrm{H}$ is a positive-definite matrix, and thus has a complete set of orthonormal eigenvectors $\left\{\boldsymbol{v}_{\ell}\right\}_{\ell=1,2, \ldots, M}$ and associated eigenvalues $\left\{\sigma_{\ell}\right\}_{\ell=1,2, \ldots, M}, \sigma_{\ell} \geq \sigma_{\ell+1} \geq 0$ that satisfy $\mathbf{H} \boldsymbol{v}_{\ell}=\sigma_{\ell} \boldsymbol{v}_{\ell}$. The quantity to be maximized in Equation 8 is a Rayleigh quotient. The quotient's maximum possible value is the largest eigenvalue $\sigma_{1}$, which is associated with the solution $v_{1}$. Finally, the synchrony of time courses is estimated by

$$
\text { Synchrony }=\sigma_{1} / \sum_{\ell=1}^{M} \sigma_{\ell} \text {. }
$$

Note that, as $\sum_{\ell=1}^{M} \sigma_{\ell}=1$ due to the normalization of the window $\mathbf{F}_{\boldsymbol{i}}(j)$, the synchrony can be simply estimated by $\sigma_{1}$, $\sigma_{1} \in[0,1]$.

\section{Data and preprocessing}

Datasets: Human MRI datasets used in this study were sourced from the WU-Minn Human Connectome Project (HCP) database ${ }^{33,34}$, which included a 128 subject sub-group $(54 \%$ female, age range $=22-37$ ) that passed image quality control criteria and completed all imaging acquisitions. The datasets comprised resting-state fMRI, motor-task fMRI, T1-weighted MRI and diffusion-weighted MRI (DWI) and were downloaded from the HCP repository as minimally preprocessed data. The HCP data acquisition was approved by the Washington University Institutional Review Board and informed consent was obtained from all subjects. Parameters of MRI acquisitions and the minimal preprocessing are fully described in reference 35. The dataset was separated into two groups: the first 64 subjects for a primary dataset and other 64 subjects for an independent validation dataset.

Preprocessing: The HCP preprocessed functional and diffusion data are in different neurological spaces (fMRI data in ACPC space; and DWI data in MNI space). Reasonably accurate co-registration of images between the two MRI modalities, which typically involves nonlinear transformations, is critical for the proposed approach. In this work, the HCP preprocessed fMRI volumes were nonlinearly registered into ACPC space, and then up-sampled to the voxel resolution of the DWI data (i.e., $1.25 \mathrm{~mm}$ isotropic). This spatial transformation was performed by leveraging the mni2acpc.nii displacement maps provided within the HCP preprocessed data. Note that the ACPC space was chosen as the working space in this study to 
avoid a spatial transformation on DWI data that would necessitate a complex adjustment of axonal orientations ${ }^{36}$. In addition, the segmentation volumes (aparc+aseg.nii) provided within the HCP data were down-sampled to the working resolution, from which a WM mask was created. Further preprocessing of fMRI data in this study included four steps as follows. First, cerebrospinal fluid (CSF) nuisance signals and 12 variates of head motion were regressed out from time courses of fMRI data. Second, the volumes of fMRI data within the WM mask were spatially smoothed with a Gaussian smoothing kernel of 3-mm full width at half maximum. Spatial smoothing this way could prevent signal contamination from nearby GM. Note that, besides improving the signal-to-noise ratio in BOLD time courses, the spatial smoothing can reduce the sensitivity of the proposed approach to potential coregistration errors at some cost of spatial specificity. Third, time courses were bandpass filtered to retain frequencies from 0.01 to $0.12 \mathrm{~Hz}$. Finally, the means of the filtered time courses were subtracted, and the data then normalized to unit variance.

\section{Synchrony mapping using FAIW-PCA}

Synchrony mapping using FAIW-PCA was performed on the HCP image data that had been co-registered in ACPC space. To begin with, a whole brain graph was constructed for each subject using the afore-mentioned approach with HARDI data. Following reference ${ }^{27}$, two tuning parameters $\{\alpha, \beta\}$ were set to $\{0.9,50\}$ in the generation of the graph so that the nature of elongated fibers can be characterized with high fidelities. With the graph constructed, the two-step activation mapping described in Sections II(A) and II(B) was performed voxelwise: (1) a local fiber-architecture-informed window was created for each voxel by the spectral kernel approach, and (2) FAIW-PCA was performed to estimate the synchrony of fMRI time-courses on the basis of fiber architecture information within the window. The parameter of the kernel $\tau$, which determines the spatial extent of signal diffusion on fibers, was optimized to ensure the distribution of the synchrony $\sigma_{1}\left(\sigma_{1} \in\right.$ $[0,1])$ in WM was peaked around 0.5 .

\section{E. Validation of FAIW-PCA-based activation mapping}

We validated the proposed approach at a group level in MNI space. To do so, synchrony maps computed from each subject in the primary dataset and the independent validation dataset were co-registered into MNI space by leveraging the acpc2mni.nii displacement maps provided within the HCP preprocessed data, and subsequently averaged to yield groupaverage synchrony maps. Then, to characterize the task-induced changes in synchrony, difference maps were calculated as

$$
\Delta \operatorname{syn}=\frac{2 \times\left(\mathrm{S}_{M}-\mathrm{S}_{R}\right)}{\mathrm{S}_{M}+\mathrm{S}_{R}},
$$

where $S_{R}$ and $S_{M}$ are the synchronies measured in resting state and motor task, respectively; values of $\Delta$ syn indicate the strength of neural responses to the task. The group-average synchrony maps and their difference maps estimated from the primary dataset were analyzed as follows: (1) the groupaverage synchrony maps from resting states were investigated first by reference to anatomical structures conveyed by $T_{1^{-}}$ weighted $\left(\mathrm{T}_{1} \mathrm{~W}\right)$ images and fractional anisotropy maps derived from the HARDI data. (2) fiber tracking was implemented in the DSI Studio software package ${ }^{37}$ to extract WM regions related to the primary motor cortex (M1) and the auditory cortex (A1\&A2); the synchrony maps from the two functional states were compared with an emphasis on the M1 related WM regions; the auditory cortex is mainly associated with processing auditory information, which is in principle not involved in motor tasks and expected to undergo minimal variations between the two functional states; thus, the specificity of the proposed approach was assessed by comparing the increase in WM activations under the motor task in the task-related and unrelated regions. (3) the regional synchronies were compared between the two functional states using the HCP tractography atlas of WM available on the website: http://brain.labsolver.org/diffusion-mritemplates/tractography. (4) group level statistical maps of $\Delta$ syn-based activation were compared with those of GLMbased activation; the statistical maps of $\Delta$ syn-based activation were derived by voxel-wise paired t-tests of differences between resting state and motor task with a false discovery rate (FDR) correction; the statistical maps of GLM-based activation were estimated using the standard SPM toolbox. Finally, comparisons of $\Delta$ syn-based activation maps between the primary dataset and the independent validation dataset were performed to validate the reproducibility of the proposed approach.

\section{Results}

Fig. 2 shows group-average synchrony maps reconstructed with the motor-task fMRI dataset collected from 64 participants in HCP, and FA color maps reconstructed with the DTI dataset from a representative participant as well as $\mathrm{T}_{1} \mathrm{w}$ images. Many continuous structures can be observed with high synchrony in both GM and WM. In the GM, the synchronous structures agree grossly with anatomical structures identified in the $\mathrm{T}_{1} \mathrm{~W}$ images. In the WM, synchronous structures exist almost exclusively in regions that contain large axonal fascicles, with some typical examples denoted by red arrows that exhibit uniform fiber orientations in the FA color maps. These spatial patterns in WM are reasonable in terms of their relationship to functional neuroanatomy. For instance, the fiber bundles in occipital WM convey mainly visual signals so that voxels within them tend to have synchronous BOLD signals. Conversely, the WM regions that include fibers with heterogenous orientations (indicated by heterogeneities in FA colors) or crossing fibers (visualized as dim FA colors) exhibit low values in the synchrony maps, which can be appreciated in the regions denoted by green arrows in Fig. 2. This is explainable by the fact that the fibers leading to different locations in GM tend to have asynchronous neural activities and thus asynchronous BOLD signals.

Fig. 3 compares the group-average synchrony maps estimated from the resting-state and motor-task fMRI data on the basis of WM tractograms, in which the motor-task-related brain activations can be visually appreciated. In Fig. 3A\&B, M1 and $\mathrm{A} 1 \& \mathrm{~A} 2$ related WM regions were determined by using fiber tracking with seed points defined in the corresponding cortical regions. It can be seen that, in both M1 GM regions and the related WM regions, the synchrony maps exhibit obviously 
bioRxiv preprint doi: https://doi.org/10.1101/2022.02.23.481698; this version posted February 24, 2022. The copyright holder for this preprint (which was not certified by peer review) is the author/funder. All rights reserved. No reuse allowed without permission.

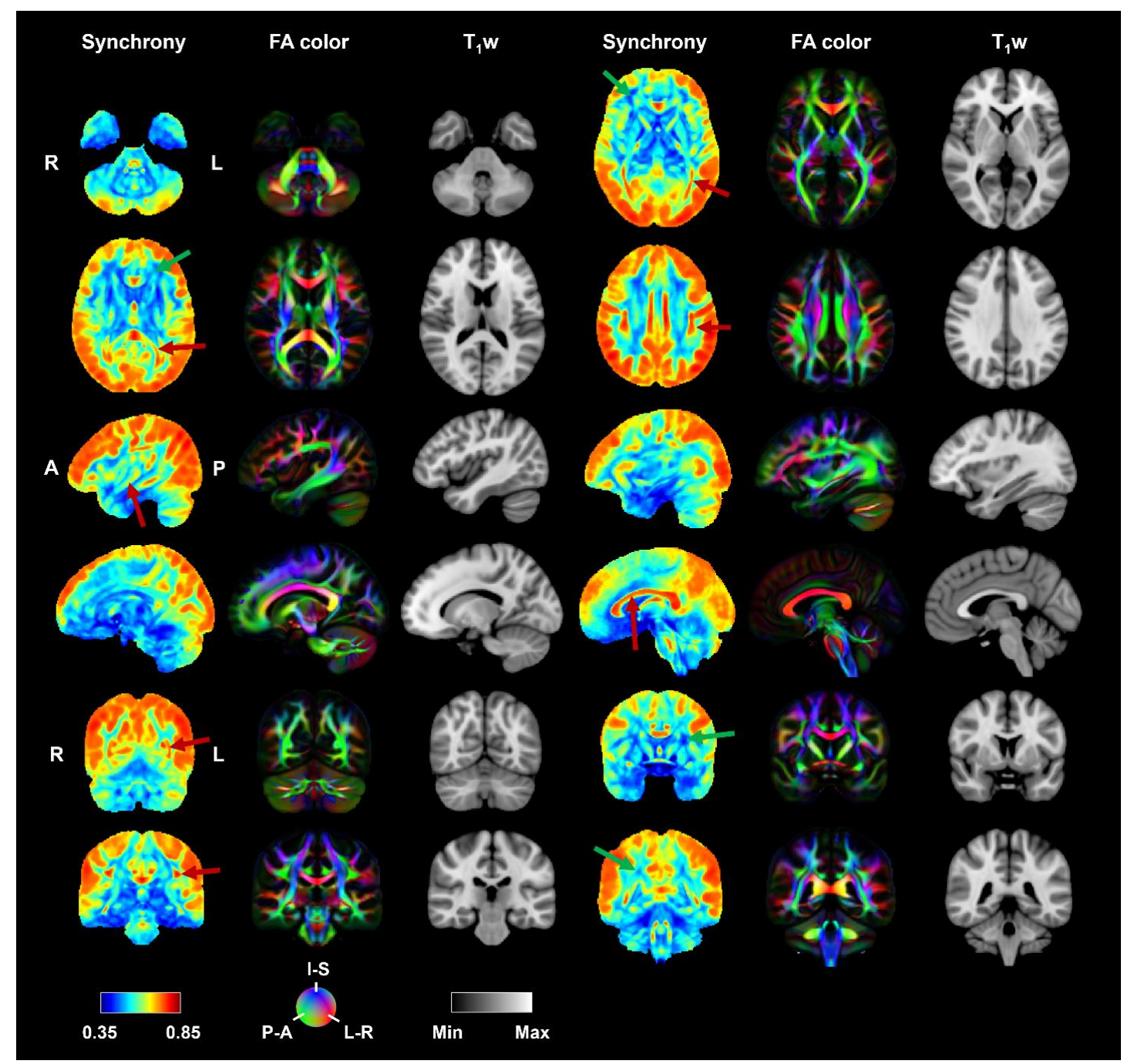

Fig.2. Group-average synchrony maps reconstructed with the motor-task fMRI dataset collected from 64 participants in HCP, and FA color maps reconstructed with the DTI dataset as well as $T_{1} w$ images from a representative participant. There are many structures with high synchrony in both GM and WM. In the GM, the synchrony structures agree grossly with anatomical structures identified in the $T_{1} w$ images. In the WM, synchrony structures exist almost exclusively in regions that contain large axonal fascicles, with some typical examples denoted by red arrows that exhibit uniform fiber orientations in the FA maps. Conversely, the WM regions that include fibers with heterogenous orientations (indicated by heterogeneities in FA colors) or crossing fibers (visualized as dim FA colors) exhibit low intensities in the synchrony maps, which is showcased in the regions denoted by green arrows. 


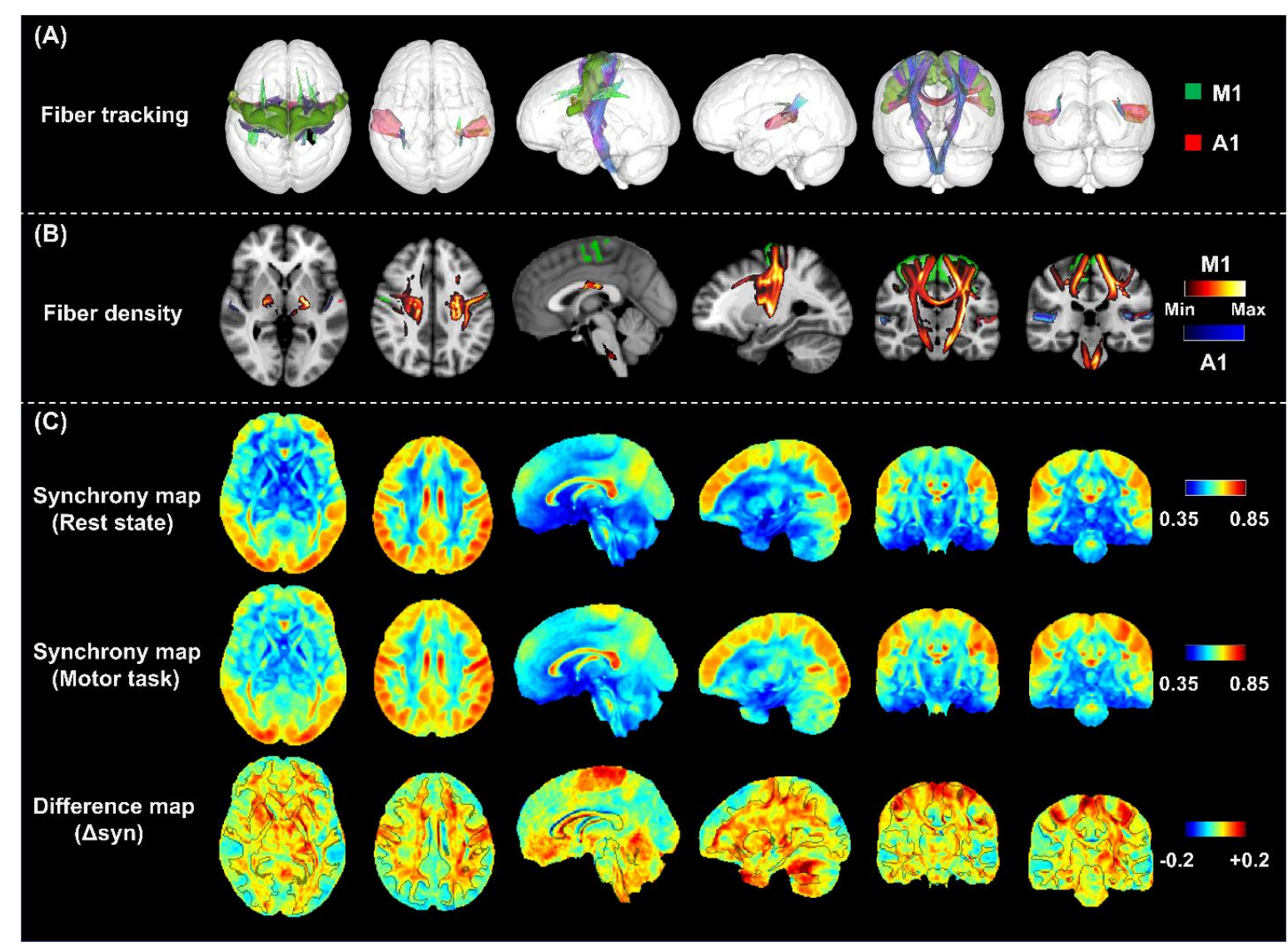

Fig. 3. Comparison of group-average activation maps obtained from resting-state fMRI data and motor-task fMRI data on the basis of WM fiber tractograms. (A), exhibition of fiber tracking. The primary motor cortex (M1) and auditory cortex (A1\&A2) related WM regions were determined by using fiber tracking with seed points defined in the corresponding cortical regions. (B), typical slices of fiber intensity maps estimated from fiber tracking. The fiber intensity of the $\mathrm{M} 1$ and $\mathrm{A} 1 \& \mathrm{~A} 2$ related WM regions are displayed with red-yellow and blue colors, respectively. (C) synchrony maps and the corresponding difference maps displayed at the same slice indexes with the images in B. In both M1 GM regions and the related WM regions, the synchrony maps exhibit obviously higher intensities under motor task than those estimated from the resting-state, which demonstrates coactivations of the associated GM and WM regions. In addition, some WM regions connected with the occipital and frontal lobes also exhibit obvious enhancements of synchrony under the motor task whereas the WM regions associated with the auditory cortex have no significant changes between the two states.

higher intensities during the motor task than in the resting-state. Coactivations of the associated GM and WM regions are quantitively visualized by the maps of synchrony difference ( $\Delta$ syn) between the two states in the bottom row of Fig. 3C. In addition, some WM regions connected with the occipital and frontal lobes also exhibit obvious enhancements of synchrony under the motor task whereas the WM regions associated with the auditory cortex have no significant changes between the two states. The changes in synchrony in M1 and A1\&A2 related WM regions are further compared in Fig. 4, in which the synchronies during the motor task are plotted against those from the resting state. The offset distances of points in the scatter plots from the identity line reflect changes in WM voxels. Evidently, the changes in BOLD signals in the M1-related region are significantly larger than those of the A1\&A2 related WM region, in which the scatter points are tightly and symmetrically distributed along the identity line.

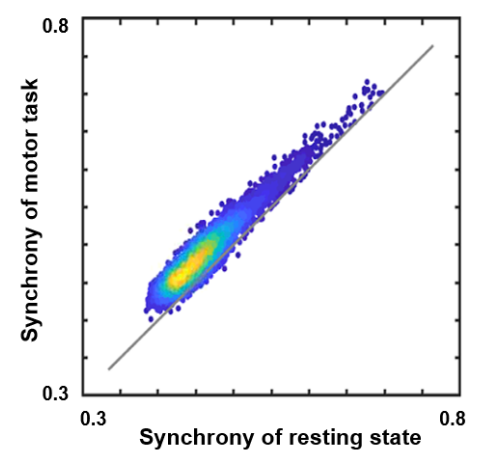

(A)

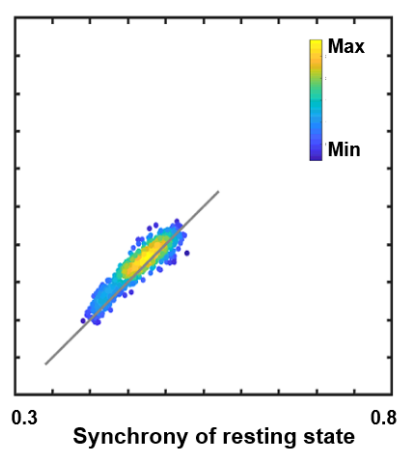

(B)
Fig. 4. Scatter pots of activations for the voxels in the primary motorcortex (M1) related WM region (A) and the auditory cortex (A1\&A2) related WM region $(B)$. Distances from points to an identity line measure the degree of activations. Activations in the M1 related WM region are significantly higher than those of the $A 1 \& A 2$ related WM region, in which the scatter points are tightly and symmetrically distributed along the identity line. 


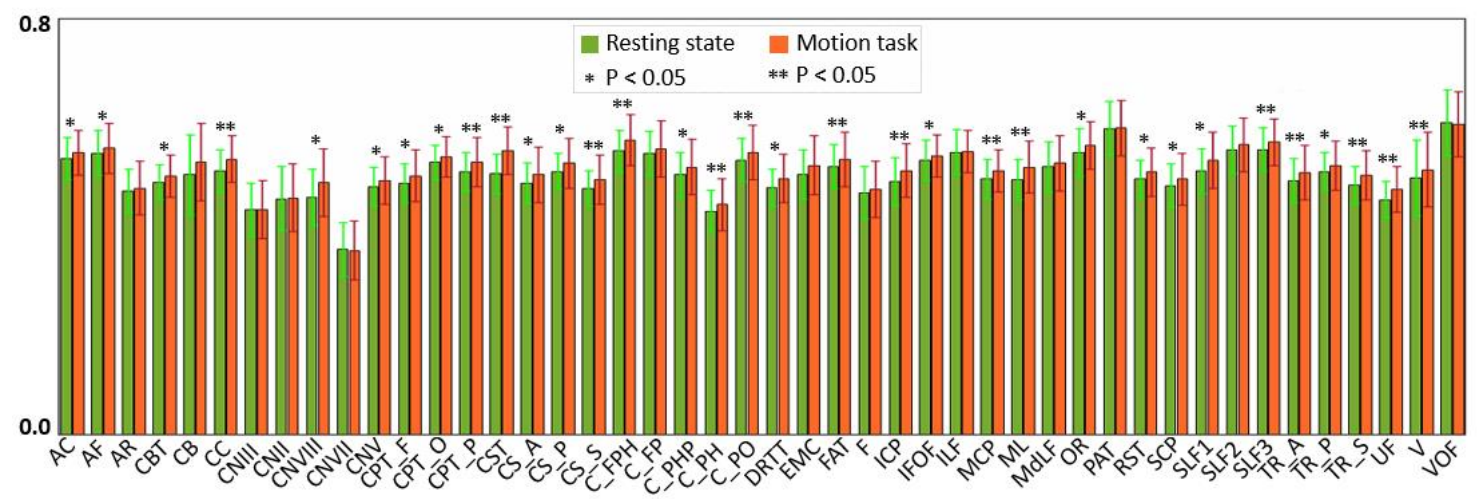

(A)

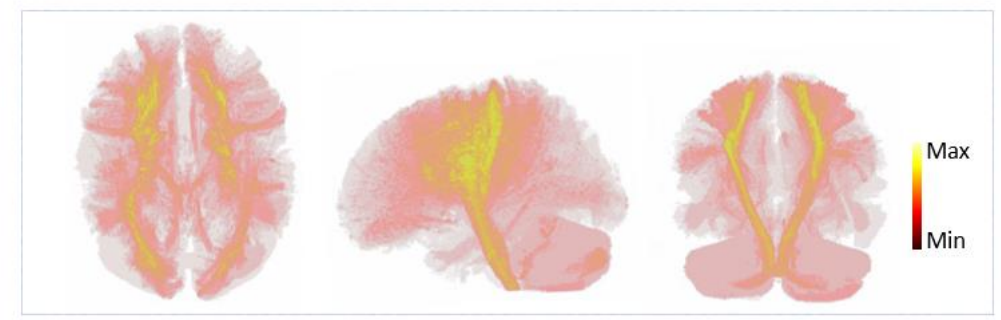

(B)

Fig. 5. Tract-wise comparison of synchrony between resting state and motor task for 46 fiber tracts defined in the HCP population-averaged tractography atlas. (A), statistical comparisons of synchrony for each fiber tract. Paired t-test was implemented with tract-averaged synchronies estimated individually from 64 subjects. The result of statistical analysis shows that the synchrony under the motor task significantly increases for 33 fiber tracts with $\mathrm{P}<0.05$, and for 16 fiber tracts with $\mathrm{P}<0.01$. (B), maximum intensity projection images of differences in tract-averaged synchronies. To visually illustrate activations of the fiber tracts, the differences in tract-averaged synchrony are assigned as intensities to the corresponding tracts with significant increases in synchrony $(P<0.05)$, which are displayed as $2 \mathrm{D}$ images using maximum intensity projections for axonal, sagittal and coronal views. High intensities in the figure appear mainly along the CST, consistent with the functional dominance of the sensorimotor pathways involved in the execution of the motor task.

In Fig. 5, tract-wise changes in synchrony from resting state to motor task are examined for 46 fiber tracts defined in the HCP population-averaged tractography atlas. Fig. 5A shows statistical comparisons for each fiber tract, where paired student tests were used to identify significant changes in tract-averaged synchronies from 64 individual subjects. As seen, the synchrony during the motor task significantly increased for 33 fiber tracts with $\mathrm{P}<0.05$, and for 16 fiber tracts with $\mathrm{P}<0.01$. Among those tracts that changed, the majority are directly engaged in sensorimotor functions or visual processing including tracts in/linking the cerebellum and brainstem (cerebellum (CB), middle cerebellar peduncle (MCP), superior cerebellar peduncle (SCP), inferior cerebellar peduncle (ICP), vermis (V) and medial lemniscus (ML) ${ }^{38,39}$, the corticospinal tract (CST) ${ }^{40}$, the corpus callosum (CC) ${ }^{41}$ and the optic radiation (OR) ${ }^{42}$. Note that many fiber tracts that are not directly involved in motor function or vision also show changes in Fig. 5A presumably because they are associated with motor network indirectly. For instance, the observed changes in the corticostriatal pathway (CS) may be explained by its role in motor planning as this fiber tract connects to the putamen, a main basal ganglia structure responsible for motor control ${ }^{43}$. Tract-wise increases in synchrony are further visually illustrated in Fig. 5B. Here, the differences in tract-averaged synchrony are assigned as intensities to the corresponding tracts with significant increases in synchrony $(\mathrm{P}<0.05)$, which are then displayed as 2D images using maximum intensity projection for axonal, sagittal and coronal views. High intensities in the figure appear mainly along the CST, consistent with the functional dominance of the sensorimotor pathways involved in the execution of the motor task.

Fig. 6 compares group level statistical maps of $\Delta$ syn-based and GLM derived changes (B), with FA color maps as anatomical references of WM (A). In the statistically inferred activation maps from both approaches, $t$-values thresholded with FDR-corrected $P$ values of 0.05 and 0.01 are shown in Fig. 6B. The GLM-indicated activation maps show that the whole M1 cortical region is engaged by the composite task, that includes foot, hand and tongue movements, which agree well with previous results ${ }^{44}$. Besides, the insular cortex that is engaged in motor control also exhibits activations in GLM statistical maps. The $\Delta$ syn-based results also highlight the cortical region of M1 with smaller coverages in the tongue motor cortex and insular cortex than the GLM-based activations. This can be actually deduced from Fig. 3 in which these regions in $\Delta$ syn-based activation maps have similarly high synchronies in both the resting state and the motor task. On the other hand, there are significant $\Delta$ syn-based changes in WM regions that are hardly captured by the GLM approach. Theses detected activations span large WM regions that are directly or indirectly associated with M1 (See the preceding paragraph). This demonstrates the power of our approach to detect functional changes in WM, though some of the detected changes in WM appear to be more disperse or discontinuous than in GM. This phenomenon may largely be attributable to inter-subject variabilities of functional structures in WM, which typically 
bioRxiv preprint doi: https://doi.org/10.1101/2022.02.23.481698; this version posted February 24, 2022. The copyright holder for this preprint

(which was not certified by peer review) is the author/funder. All rights reserved. No reuse allowed without permission.

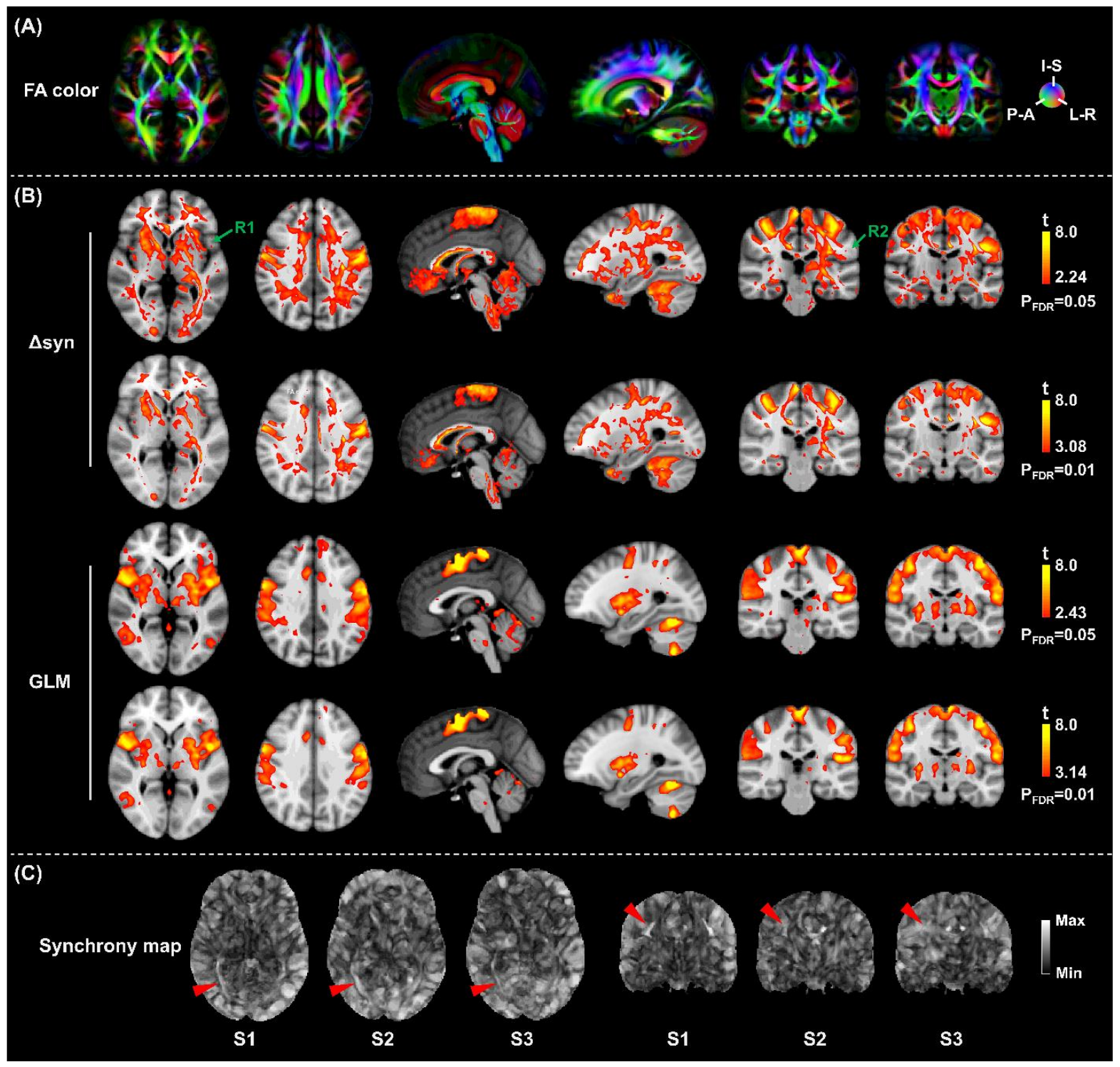

Fig. 6. Comparison of group level statistical maps of $\Delta$ syn- and GLM-based activation with anatomical references of fiber structures. (A), FA color maps from one representative participant. The FA color maps show fiber structures. (B), group level statistical maps of $\Delta$ syn- and GLM-based activation. The statistical maps of $\Delta$ syn-based changes are derived by voxel-wise paired t-test of differences between resting state and motor task with a false discovery rate (FDR) correction for the 64-subject dataset. The statistical maps of GLM-based activation are estimated by using the standard SPM toolbox. In the statistically inferred activation maps from both the approaches, $t$-values thresholded with the FDR-corrected $P$ values of 0.05 and 0.01 are shown in B. The GLM-based activation maps indicate that the whole primary motor cortex (M1) is engaged by the composite task that includes foot, hand and tongue movements. The $\Delta$ syn-based activations also highlight the cortical region of M1 and show smaller coverages in the insular cortex (R1) and the tongue motor cortex (R2) than the GLM-indicated activations. Moreover, the $\Delta$ syn-based activations exhibit in WM regions that are hardly captured by the GLM approach. The green arrows indicate the activations in WM that have similar spatial patterns with fiber structures identified in FA color maps. (C), synchrony maps of three typical subjects displayed in MNI space. There are discernable inter-subject variabilities of elongated fibers (indicated by red arrows) among these subjects, which could confound the statistical analysis at group level, and thus may account for the dispersity and discontinuity of activations detected in WM. 


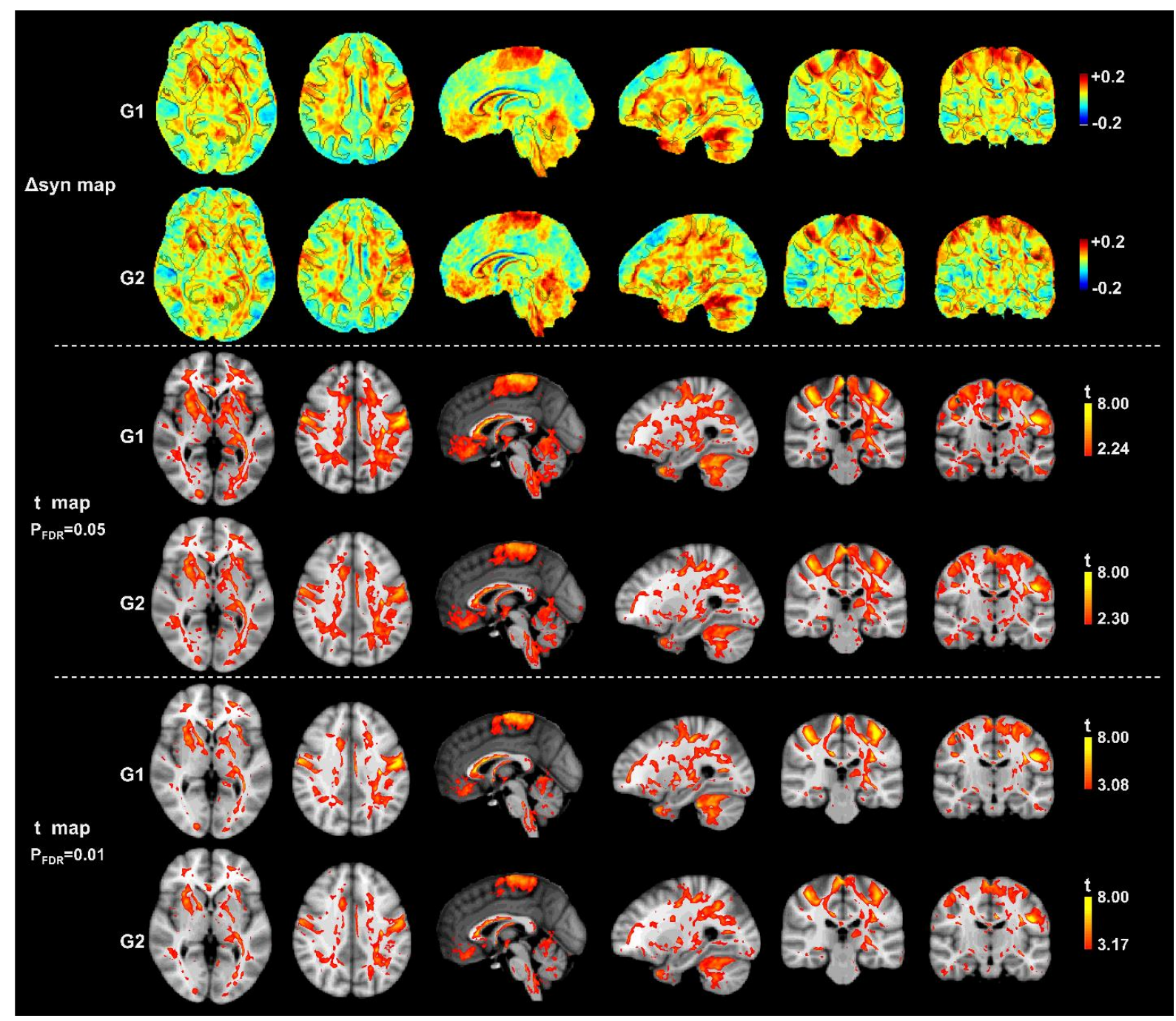

Fig. 7. The reproducibility of the FAIW-PCA activation mapping validated with two independent datasets (G1 and G2). (A), group-average $\Delta$ syn maps estimated from the two datasets. The two maps exhibit similar spatial patterns of the task-induced enhancement of synchrony. (B\&C), statistical t maps of $\Delta$ syn-based activation from the two datasets. In B\&C, t values are thresholded with the FDR-corrected P values of 0.05 and 0.01 , respectively. Dice coefficient is calculated to quantify the similarity between the activation maps from the two datasets. Dice coefficients of the activation maps estimated for $B$ and $C$ are 0.79 and 0.84 , respectively, which demonstrates a good reproducibility of the proposed approach.

have elongated geometry, thus tending to confound group level statistical analyses due to imperfect registrations. These spatial variabilities are demonstrated by the synchrony structures denoted by the red arrows in Fig. 6C, which shows synchrony maps of three typical subjects that have been registered into MNI space. In spite of the use of fine nonlinear registrations, elongated subtle synchrony structures in WM show discernable spatial variations among these subjects.

Fig. 7 demonstrates the reproducibility of the FAIW-PCA activation mapping, which is validated by two independent datasets (G1 and G2). The group-average $\Delta$ syn maps estimated from the two datasets are displayed in Fig. 7A, which show very similar spatial patterns of the enhancement of synchrony by the task. Figs. 7B\&C show statistical $\mathrm{t}$ maps of $\Delta$ syn-based activations for the two datasets, which are thresholded with FDR-corrected $\mathrm{P}$ values of 0.05 and 0.01 . To quantify the similarity between the activation maps from the two datasets, the Dice coefficient was calculated as

$$
D=\frac{2\left|M_{G 1} \cap M_{G 2}\right|}{\left|M_{G 1}\right|+\left|M_{G 2}\right|}
$$

where $M_{G 1}$ and $M_{G 2}$ are two sets of activated voxels detected from G1 and G2, respectively and $|\cdot|$ denotes set cardinality. The Dice coefficient is constrained to the range of $[0,1]$, where a value 1 signifies perfect overlap between the two maps and a value 0 represents no overlap. In this study, Dice coefficients of the activation maps in $\mathrm{B}$ and $\mathrm{C}$ are 0.79 and 0.84 , respectively, which demonstrates a high reproducibility of the FAIW-PCA activation mapping.

\section{Discussion}

Localizing neural activations under specific functional loadings is fundamental to understanding the functional organization of the brain. GLM-based activation mapping has been the standard approach for detecting and localizing task- 
evoked activations in GM, and assumes that BOLD signals in response to neural activation can be modeled by convolving the time course of neural activity with a fixed HRF that is determined a priori ${ }^{45}$. However, this approach is inappropriate for WM. In this study, we proposed a model-free approach to detect changes in BOLD signals associated with neural activities in WM by measuring the enhancement of synchrony in WM BOLD signals during a functional task. The model-free approach draws upon an assumption that, under functional loadings, BOLD signals in relevant WM fibers are modulated by stimulus-evoked neural responses and thereby become more synchronized than in a resting state. This approach consists of two major steps. First, a fiber-architecture-informed spatial window is created with ODFs constructed from HARDI data, which characterizes a topographically defined neighborhood of a voxel that is isomorphic to local fiber architectures within WM. Second, a modified PCA is used to estimate the synchrony of BOLD signals based on the spatial window. The proposed approach is validated using a cohort of $3 \mathrm{~T}$ fMRI datasets from the HCP, which demonstrates that the changes associated with neural activation can be reliably detected as enhancements of fMRI signal synchrony in the fibers that are engaged in the presented task.

Structures defined by intrinsic synchronous signals exist in both GM and WM and have similar boundaries with other definitions of anatomical and functional structures (Fig. 2). For GM, they appear as separable volumes with low degrees of anisotropy that are consistent with identifiable functional areas or units that to date have been implicitly assumed in the study of the functional connectome (i.e., Brodmann areas). In contrast to GM, functional units of WM measured by fMRI are elongated fibers that facilitate the transmission of neural signals along the fibers, which are identifiable by WM synchronous structures shown in Fig. 2 and Fig. 5C at group and individual levels, respectively. These synchronous components can be clearly observed in a resting state and become more pronounced in functionally-relevant structures under task loading (Fig. 3), which agrees with previous findings that correlations of BOLD signals in relevant WM bundles increase with tasks ${ }^{24-26}$. Note that the regions that contain parallel fascicles tend to have higher levels of synchrony than those with incoherent orientations because some fibers in the latter scenario are in principle connected to different functional units in GM such that synchronous BOLD signals are not apparent.

By comparing group-average synchrony maps derived from resting-state and motor-task fMRI data, we demonstrate that BOLD signal synchrony can be enhanced by task loading in both GM and WM (Fig. 3, 4 and 5). For the GM, group level statistical maps of $\Delta$ syn-based activations in the M1 region show a moderate overlap with those of GLM-based activations, although with a smaller coverage than the latter, especially in the tongue motor cortex and the insular cortex (Fig. 6). The missing voxels of $\Delta$ syn-based activations in the tongue motor cortex may be explained by subconscious swallowing activity that inevitably occurs during the resting state acquisitions, which is implied by the observation in Fig. 3 that the missing regions in $\Delta$ syn-based activation maps have similar synchronies in both the resting state and the motor task. Likewise, the insular cortex, which possesses multiple functions such as interoceptive attention, multimodal sensory processing, autonomic control and perceptual self-awareness ${ }^{46,47}$, may be equally activate in the two states, and thus show similar synchronies. The $\Delta$ syn maps exhibit large increases in the M1related WM regions in Fig. 3, which demonstrates that $\Delta$ synbased activation mapping possesses a distinctive capability to detect activity-related signal changes in WM that are not captured by the GLM approach. This highlights the possibility that changes in synchrony do not depend on net increases in BOLD signal. In addition, some WM regions leading to the occipital and frontal lobes also exhibit obvious enhancements of synchrony under the motor task in Fig. 3. The spatial patterns of synchrony increases are consistent with previous reports that the occipital and frontal lobes are respectively engaged in visual processing ${ }^{48,49}$ and executive functions ${ }^{50}$ associated with the motor task. Further statistical analysis based on a populationaveraged tractography atlas demonstrate that many fiber tracts that are indirectly associated with M1 also show significant enhancements of BOLD signal synchrony (Fig. 5). This implies a massive engagement of WM in the execution of the motor task, which agrees with earlier reports that GM across almost the entire brain can be activated by a simple task. Although many WM regions are involved in the motor task ${ }^{51}$, the M1-related WM exhibit most significant changes, as shown Fig. 5B.

In this study, the sensitivity, specificity and reproducibility of the proposed approach have been comprehensively investigated. First, the $\Delta$ syn-based activations can be clearly observed in the M1-related WM regions, suggesting this approach has reasonable sensitivity. It is well-known that the magnitudes and signal-to-noise ratios of BOLD signals in WM are substantially lower than those in GM [19] due to the significantly lower blood volume in WM compared to that in GM [1, 20], so detecting changes in the magnitudes of BOLD signals in a task is challenging. Alternatively, our model-free approach exploits the enhancements of BOLD signal synchrony to identify neural activations, which is robust in the presence of noise without modeling the BOLD signal. Second, the specificity of the proposed approach is only partially validated by the observation that there are no significant enhancements of synchrony in auditory cortex under the motor task (Fig. 4B). We found that some other WM regions that are not expected to be directly associated with the task targeted M1 region also show significant enhancements of synchrony, though these may be indirectly affected. Third, the reproducibility of the proposed approach was validated using two independent groups of participants where statistical $t$ maps of $\Delta$ syn-based activation thresholded with the FDR-corrected P values of 0.05 and 0.01 were compared. Dice coefficients of the activation maps estimated from the two groups are 0.79 and 0.84 , respectively, which demonstrates high reproducibility of the FAIW-PCA activation mapping.

To validate the proposed approach with large-sample datasets, we analyzed the open-access HCP datasets of 128 participants, though the parameters of MRI acquisition and the designs of the fMRI tasks were not tailored for our study, which thus led to some limitations. First, parameters of MRI acquisitions in the HCP data are optimized for fMRI of GM. In a future study, the parameters of MRI acquisitions can be optimized for detecting synchrony of BOLD signals in WM, which may not require high temporal resolution. Second, the motor task of the HCP involves five different motions that 
produce only about 18 data points in each time courses for each motion. Some regions in brain that are engaged by only one motion may be more difficult to be detected by our approach because the measurement of the synchrony is sensitive to the number of data points in time courses. This limitation in the task design can reduce the sensitivity of the proposed approach, which may be one reason for the loss of activations detected compared to the GLM results. In a future study, increasing the data points that are modulated by identical repeated stimuli can be used to increase the sensitivity of the proposed method.

\section{Conclusions}

The GLM-based activation mapping that is well-established for detecting BOLD signal changes in a task in GM is not well suited for studies of activity-related signal changes in WM. In this study, we proposed a model-free approach to detect taskevoked signal changes in WM by measuring increases in BOLD signal synchrony within local fiber architectures. Using the HCP datasets, we validated that the proposed approach could detect BOLD signal changes in WM fibers that engaged in the motor task with high sensitivities and reproducibility. This approach promises to provide a standardized tool to map neural responses in WM in the future study.

\section{Declaration of competing interests}

The authors declare that they have no competing interests.

\section{Code and data availability}

An implementation of the approach proposed in this work will be made available as a MATLAB package on GitHub. Customized links will be added in the final version of the manuscript. Human MRI datasets used in this study were sourced from the WU-Minn Human Connectome Project (HCP) database.

\section{Acknowledgements}

Data used in this work were provided by the Human Connectome Project, WU-Minn Consortium (Principal Investigators: David Van Essen and Kamil Ugurbil; 1U54MH091657) funded by the 16 Institutes and Centers of the National Institutes of Health (NIH) that support the NIH Blueprint for Neuroscience Research; and by the McDonnell Center for Systems Neuroscience at Washington University. This work was supported by National Institutes of Health under Grant R01NS093669 and Grant R01NS113832.

\section{References}

1 Gawryluk, J. R., Mazerolle, E. L. \& D'Arcy, R. C. Does functional MRI detect activation in white matter? A review of emerging evidence, issues, and future directions. Front Neurosci 8, 239, doi:10.3389/fnins.2014.00239 (2014).

2 Mazerolle, E. L., Ohlhauser, L., Mayo, C. D., Sheriff, A. \& Gawryluk, J. R. Evidence of Underreporting of White Matter fMRI Activation. $J$ Magn Reson Imaging 51, 1596-1597, doi:10.1002/jmri.26952 (2020).

3 Gore, J. C. et al. Functional MRI and resting state connectivity in white matter - a mini-review. Magn Reson Imaging 63, 1-11, doi:10.1016/j.mri.2019.07.017 (2019).

4 Grajauskas, L. A., Frizzell, T., Song, X. \& D'Arcy, R. C. N. White Matter fMRI Activation Cannot $\mathrm{Be}$ Treated as a Nuisance Regressor: Overcoming a Historical Blind Spot. Front Neurosci 13, 1024, doi:10.3389/fnins.2019.01024 (2019).

5 Ding, Z. et al. Detection of synchronous brain activity in white matter tracts at rest and under functional loading. Proc Natl Acad Sci U S A 115, 595-600, doi:10.1073/pnas.1711567115 (2018).

Gao, Y. et al. Lower functional connectivity of white matter during rest and working memory tasks is associated with cognitive impairments in schizophrenia. Schizophr Res 233, 101-110, doi:10.1016/j.schres.2021.06.013 (2021).

7 Jiang, Y. et al. White-matter functional networks changes in patients with schizophrenia. Neuroimage 190, 172-181, doi:10.1016/j.neuroimage.2018.04.018 (2019).

8 Huang, Y. et al. Voxel-wise detection of functional networks in white matter. Neuroimage 183, 544-552, doi:10.1016/j.neuroimage.2018.08.049 (2018).

9 Marussich, L., Lu, K. H., Wen, H. \& Liu, Z. Mapping white-matter functional organization at rest and during naturalistic visual perception. Neuroimage 146, 1128-1141, doi:10.1016/j.neuroimage.2016.10.005 (2017).

10 Ji, G. J. et al. Regional and network properties of white matter function in Parkinson's disease. Hum Brain Mapp 40, 1253-1263, doi:10.1002/hbm.24444 (2019).

11 Li, M., Gao, Y., Ding, Z. \& Gore, J. C. Power spectra reveal distinct BOLD resting-state time courses in white matter. Proceedings of the National Academy of Sciences 118, doi:10.1073/pnas.2103104118 (2021).

12 Li, M., Newton, A. T., Anderson, A. W., Ding, Z. \& Gore, J. C. Characterization of the hemodynamic response function in white matter tracts for event-related fMRI. Nat Commun 10, 1140, doi:10.1038/s41467-019-09076-2 (2019).

13 Poline, J. B. \& Brett, M. The general linear model and fMRI: does love last forever? Neuroimage 62, 871-880, doi:10.1016/j.neuroimage.2012.01.133 (2012).

14 Zhang, D. \& Raichle, M. E. Disease and the brain's dark energy. Nat Rev Neurol 6, 15-28, doi:10.1038/nrneurol.2009.198 (2010).

15 Laird, A. R. et al. Behavioral interpretations of intrinsic connectivity networks. $J \quad$ Cogn Neurosci 23, 4022-4037, doi:10.1162/jocn_a_00077 (2011).

16 Schilling, K. G. et al. Histological validation of diffusion MRI fiber orientation distributions and dispersion. Neuroimage 165, 200-221, doi:10.1016/j.neuroimage.2017.10.046 (2018).

17 Voigt, F. F. et al. The mesoSPIM initiative: open-source light-sheet microscopes for imaging cleared tissue. Nat Methods 16, 11051108, doi:10.1038/s41592-019-0554-0 (2019).

18 Courtemanche, M. J., Sparrey, C. J., Song, X., MacKay, A. \& D'Arcy, R. C. N. Detecting white matter activity using conventional 3 Tesla fMRI: An evaluation of standard field strength and hemodynamic response function. Neuroimage 169, 145-150, doi:10.1016/j.neuroimage.2017.12.008 (2018).

19 Yarkoni, T., Barch, D. M., Gray, J. R., Conturo, T. E. \& Braver, T. $\mathrm{S}$. BOLD correlates of trial-by-trial reaction time variability in gray and white matter: a multi-study fMRI analysis. PLoS One 4, e4257, doi:10.1371/journal.pone.0004257 (2009).

20 Jochimsen, T. H. et al. Whole-brain mapping of venous vessel size in humans using the hypercapnia-induced BOLD effect. Neuroimage 51, 765-774, doi:10.1016/j.neuroimage.2010.02.037 (2010).

21 Zang, Y., Jiang, T., Lu, Y., He, Y. \& Tian, L. Regional homogeneity approach to fMRI data analysis. Neuroimage 22, 394-400, doi:10.1016/j.neuroimage.2003.12.030 (2004).

22 Yuan, R. et al. Regional homogeneity of resting-state fMRI contributes to both neurovascular and task activation variations. Magn Reson Imaging 31, 1492-1500, doi:10.1016/j.mri.2013.07.005 (2013). 
bioRxiv preprint doi: https://doi.org/10.1101/2022.02.23.481698; this version posted February 24, 2022. The copyright holder for this preprint (which was not certified by peer review) is the author/funder. All rights reserved. No reuse allowed without permission.

23 Lv, Y., Margulies, D. S., Villringer, A. \& Zang, Y. F. Effects of finger tapping frequency on regional homogeneity of sensorimotor cortex. PLoS One 8, e64115, doi:10.1371/journal.pone.0064115 (2013).

Ding, Z. et al. Spatio-temporal correlation tensors reveal functional structure in human brain. PLoS One 8, e82107, doi:10.1371/journal.pone.0082107 (2013).

Ding, Z. et al. Visualizing functional pathways in the human brain using correlation tensors and magnetic resonance imaging. Magn Reson Imaging 34, 8-17, doi:10.1016/j.mri.2015.10.003 (2016). Schilling, K. G. et al. Functional tractography of white matter by high angular resolution functional-correlation imaging (HARFI). Magn Reson Med 81, 2011-2024, doi:10.1002/mrm.27512 (2019). Abramian, D. et al. Diffusion-informed spatial smoothing of fMRI data in white matter using spectral graph filters. Neuroimage 237, 118095, doi:10.1016/j.neuroimage.2021.118095 (2021).

Shuman, D. I., Narang, S. K., Frossard, P., Ortega, A. \& Vandergheynst, P. The emerging field of signal processing on graphs: Extending high-dimensional data analysis to networks and other irregular domains. IEEE Signal Processing Magazine 30, 8398, doi:10.1109/msp.2012.2235192 (2013).

Ortega, A., Frossard, P., Kovacevic, J., Moura, J. M. F. \& Vandergheynst, P. Graph Signal Processing: Overview, Challenges, and Applications. Proceedings of the IEEE 106, 808-828, doi:10.1109/jproc.2018.2820126 (2018).

Iturria-Medina, Y. et al. Characterizing brain anatomical connections using diffusion weighted MRI and graph theory. Neuroimage 36, 645-660, doi:10.1016/j.neuroimage.2007.02.012 (2007).

Turner, R. How much cortex can a vein drain? Downstream dilution of activation-related cerebral blood oxygenation changes. Neuroimage 16, 1062-1067, doi:10.1006/nimg.2002.1082 (2002).

Tian, Y., Margulies, D. S., Breakspear, M. \& Zalesky, A. Topographic organization of the human subcortex unveiled with functional connectivity gradients. Nat Neurosci 23, 1421-1432, doi:10.1038/s41593-020-00711-6 (2020).

Van Essen, D. C. et al. The WU-Minn Human Connectome Project: an overview. Neuroimage $82-79$, doi:10.1016/j.neuroimage.2013.05.041 (2013).

Smith, S. M. et al. Resting-state fMRI in the Human Connectome Project. Neuroimage 80, 144-168, doi:10.1016/j.neuroimage.2013.05.039 (2013).

Glasser, M. F. et al. The minimal preprocessing pipelines for the Human Connectome Project. Neuroimage 80, 105-124, doi:10.1016/j.neuroimage.2013.04.127 (2013).

Alexander, D. C., Pierpaoli, C., Basser, P. J. \& Gee, J. C. Spatial transformations of diffusion tensor magnetic resonance images. IEEE Trans Med Imaging 20, 1131-1139, doi:10.1109/42.963816 (2001).

Yeh, F. C., Wedeen, V. J. \& Tseng, W. Y. Generalized q-sampling imaging. IEEE Trans Med Imaging 29, 1626-1635, doi:10.1109/TMI.2010.2045126 (2010).

Catani, M. \& Thiebaut de Schotten, M. A diffusion tensor imaging tractography atlas for virtual in vivo dissections. Cortex 44, 11051132, doi:10.1016/j.cortex.2008.05.004 (2008).

Dell'Acqua, F., Bodi, I., Slater, D., Catani, M. \& Modo, M. MR diffusion histology and micro-tractography reveal mesoscale features of the human cerebellum. Cerebellum 12, 923-931, doi:10.1007/s12311-013-0503-x (2013).

Cho, S. H. et al. Motor outcome according to the integrity of the corticospinal tract determined by diffusion tensor tractography in the early stage of corona radiata infarct. Neurosci Lett 426, 123-127, doi:10.1016/j.neulet.2007.08.049 (2007).

Saenz, M. \& Fine, I. Topographic organization of V1 projections through the corpus callosum in humans. Neuroimage 52, 12241229, doi:10.1016/j.neuroimage.2010.05.060 (2010).

Hofer, S., Karaus, A. \& Frahm, J. Reconstruction and dissection of the entire human visual pathway using diffusion tensor MRI. Front Neuroanat 4, 15, doi:10.3389/fnana.2010.00015 (2010).

Haber, S. N. Corticostriatal circuitry. Dialogues Clin Neurosci 18, 7-21 (2016).

Barch, D. M. et al. Function in the human connectome: task-fMRI and individual differences in behavior. Neuroimage 80, 169-189, doi:10.1016/j.neuroimage.2013.05.033 (2013).
Boynton, G. M., Engel, S. A. \& Heeger, D. J. Linear systems analysis of the fMRI signal. Neuroimage 62, 975-984, doi:10.1016/j.neuroimage.2012.01.082 (2012).

Nieuwenhuys, R. The insular cortex: a review. Prog Brain Res 195, 123-163, doi:10.1016/B978-0-444-53860-4.00007-6 (2012).

Benarroch, E. E. Insular cortex: Functional complexity and clinical correlations. Neurology 93, 932-938, doi:10.1212/WNL.0000000000008525 (2019).

48 Duffau, H. Stimulation mapping of white matter tracts to study brain functional connectivity. Nat Rev Neurol 11, 255-265, doi:10.1038/nrneurol.2015.51 (2015).

49 Andrews, T. J., Halpern, S. D. \& Purves, D. Correlated size variations in human visual cortex, lateral geniculate nucleus, and optic tract. $J$ Neurosci 17, 2859-2868 (1997).

50 Badre, D. \& Desrochers, T. M. Hierarchical cognitive control and the frontal lobes. Handb Clin Neurol 163, 165-177, doi:10.1016/B978-0-12-804281-6.00009-4 (2019).

51 Gonzalez-Castillo, J. et al. Whole-brain, time-locked activation with simple tasks revealed using massive averaging and model-free analysis. Proc Natl Acad Sci U S A 109, 5487-5492, doi:10.1073/pnas.1121049109 (2012). 